\title{
ROOTS OF UNITY IN DEFINITE QUATERNION ORDERS
}

\author{
LUIS ARENAS-CARMONA
}

\begin{abstract}
A commutative order in a quaternion algebra is called selective if it is embeds into some, but not all, the maximal orders in the algebra. It is known that a given quadratic order over a number field can be selective in at most one indefinite quaternion algebra. Here we prove that the order generated by a cubic root of unity is selective for any definite quaternion algebra over the rationals with a type number 3 or larger. The proof extends to a few other closely related orders.
\end{abstract}

\section{INTRODUCTION}

A commutative order $\mathfrak{H}$ in a quaternion algebra $\mathfrak{A}$, over a finite field $K$, is said to be selective if it is contained in some, but not all, of the maximal orders in the algebra. More generally, for a genus $\mathbb{O}$ of orders of maximal rank in $\mathfrak{A}$, a commutative order $\mathfrak{H}$ is called selective for $\mathbb{O}$ if it embeds in some orders in $\mathbb{O}$, but not in all of them. The selective orders, for the genus of maximal order in a quaternion algebra, were characterized by Friedman and Chindburg [11, provided that the algebra satisfied Eichler's condition, namely:

For any finite set $T$ of places of $K$ containing the set $\infty(K)$ of archimedean places, Eichler's condition for $T$ is satisfied if there exist a place $\wp \in T$ such that $\mathfrak{A}_{\wp}$ is a matrix algebra. If $T$ is omitted, it is assumed that $T=\infty(K)$.

Later several authors extended this characterization to Eichler orders [12], [7. B. Linowitz has given several criteria under which selectivity can be avoided, always assuming Eichler's condition [15]. All these result are based on the fact that, if Eichler's condition holds, every spinor genus of orders of maximal rank in $K$ contains a unique conjugacy class, and representations of orders by spinor genera can be studied by purely local computations, using the machinery of class field theory. In fact, the proportion of spinor genera representing a given suborder (commutative or not), is frequently a rational number of the form $1 /[F: K]$, 
for some explicit class field $F$, the representation field. It is often the case that $F \subseteq L$ when $\mathfrak{H}$ is a suborder contained on a subfield $L$ of $\mathfrak{A}$. This shows that, for any such order $\mathfrak{H}$, the proportion of spinor genera in a genus representing $\mathfrak{H}$ is 0,1 , or $1 / 2$. The field $F$ is also contained in the spinor class field of the genus [1]. This shows that, if the Eichler's condition is satisfied, for any given genus only a finite number of subfields can contain selective orders. On the other hand, we showed in [4] that any given commutative order $\mathfrak{H}$ can be selective in some genera of at most one quaternion algebra $\mathfrak{A}(\mathfrak{H})$. If $\mathfrak{H}$ spans the field $L=K(\sqrt{d})$, then $\mathfrak{A}(\mathfrak{H})$ is defined by the Hilbert symbol

$$
\mathfrak{A}(\mathfrak{H})=\left(\frac{d,-1}{K}\right) .
$$

The preceding discussion implies that, when Eichler's condition is satisfied, selectivity is a rare phenomenon. The purpose of this work is to provide some evidence that this might not be so for quaternion algebras failing to satisfy Eichler's condition, i.e., definite quaternion algebras. Here we concentrate mostly on the case where $K=\mathbb{Q}$ and $\mathfrak{H}=\mathbb{Z}[\omega]$, where $\omega$ is a primitive cubic root of unity, but this is due to the fact that this specific order has a particularly nice characterization in terms of quotient graphs.

Theorem 1.1. Let $(\mathbb{O}$ be a genus of Eichler orders of odd level in a definite rational quaternion algebra $\mathfrak{A}$ that is unramified at 2 . Assume that $\left(10\right.$ contains $n$ conjugacy classes of orders, then at most $\frac{n}{2}+1$ of them contain cubic roots of unity.

Corollary 1.1. Let $\mathbb{O}$ be a genus of Eichler orders in a definite rational quaternion algebra $\mathfrak{A}$ that is unramified at 2 . Then if $\mathbb{Z}[\omega]$ is represented by $\mathbb{O}$, it is selective for $\mathbb{O}$, as soon as $\mathbb{O}$ contains at least three conjugacy classes.

Note that the preceding corollary does not assume an odd level for the Eichler order. This is only due to the fact that $\omega$ generates an unramified quadratic extension of $\mathbb{Q}_{2}$, and therefore it is contained in a unique maximal order at 2 [5, Prop. 4.2], so an Eichler order of even level cannot contain a copy of $\mathbb{Z}[\omega]$. The preceding result does not mention spinor genera, since every genus of Eichler orders in a quaternion algebra over $\mathbb{Q}$ has a unique spinor genus. This is not the case for more general orders.

Theorem 1.2. Let (1) be a spinor genus of orders of maximal rank in a definite rational quaternion algebra $\mathfrak{A}$ that is unramified at 2 . Assume 
that the orders of $\mathbb{O}$ are maximal at 2 . Then, if $|\mathbb{O}|=n$, at most $\frac{3}{4}(n+1)$ orders in $\mathbb{O}$ contain cubic roots of unity.

Corollary 1.2. Let $\mathbb{O}$ be a spinor genus of orders of maximal rank in a definite rational quaternion algebra $\mathfrak{A}$ that is unramified at 2 . Assume that the orders of $\mathbb{O}$ are maximal at 2 . Then if $\mathbb{Z}[\omega]$ is represented by $\mathbb{O}$, it is selective for $\mathbb{O}$, as soon as $\mathbb{O}$ contains at least four conjugacy classes.

The latter result can be refined a little more using the theory of representation by spinor genera. An order is called spinor selective for a given genus if it is represented by some, but not all, the spinor genera in that genus.

Theorem 1.3. Let $(\mathbb{O}$ be a spinor genus of orders of maximal rank in a definite rational quaternion algebra $\mathfrak{A}$ satisfying the following conditions:

- $\mathfrak{A}$ is unramified at 2 ,

- the orders in $\mathbb{O}$ are maximal at 2 ,

- (O) contains 3 conjugacy classes, and

- $\mathbb{Z}[\omega]$ is not spinor selective for the genus $\hat{\mathbb{O}}$ containing $\mathbb{O}$.

Then $\mathbb{Z}[\omega]$ is selective for $\mathbb{O}$.

\section{AdELES AND SPINOR GENERA}

Let $K$ be a number field, and let $\mathfrak{A}$ be a quaternion $K$-algebra. For every place $\wp$ of $K$, we let $K_{\wp}$ and $\mathfrak{A}_{\wp}$ be the corresponding completions. We let $\mathbb{A}=\mathbb{A}_{K}$ be the adele ring on $K$, namely the direct limit

$$
\mathbb{A}_{K}=\lim _{\longrightarrow} \mathcal{O}_{\mathbb{A}, T}, \quad \mathcal{O}_{\mathbb{A}, T}=\left(\prod_{\wp \in T} K_{\wp}\right) \times\left(\prod_{\wp \in \Pi(K)-T} \mathcal{O}_{\wp}\right),
$$

where $\Pi(K)$ is the set of all places of $K, \mathcal{O}_{\wp}$ is the ring of integers of the local field $K_{\wp}$, and the direct limit is taken with respect to the directed set of finite subsets $T$ of $\Pi(K)$ containing $\infty(K)$. This defines a topology on the ring $\mathbb{A}$. Similarly, if $\mathfrak{D}$ is an order of maximal rank in $\mathfrak{A}$, we define, for every finite set $T \subseteq \Pi(K)$ containing $\infty(K)$, the $T$-adelization

$$
\mathfrak{D}_{\mathbb{A}, T}=\left(\prod_{\wp \in T} \mathfrak{A}_{\wp}\right) \times\left(\prod_{\wp \in \Pi(K)-T} \mathfrak{D}_{\wp}\right) .
$$

The adelization $\mathfrak{A}_{\mathbb{A}}$ is defined as the direct limit of the rings $\mathfrak{D}_{\mathbb{A}, T}$. Note that this definition is independent of the choice of $\mathfrak{D}$ since two 
lattices on a $K$-vector space coincide at almost all places. The ring $\mathbb{A}$ is naturally identified with the sub-ring of $\prod_{\wp \in \Pi(K)} K_{\wp}$ whose coordinates are integral at almost all places, although the topology of $\mathbb{A}$ as a direct limit is strictly stronger than the product topology. The same observations apply to $\mathfrak{A}_{\mathbb{A}}$. For any order $\mathfrak{D}$, and any finite set of places $T$ containing $\infty(K)$, the ring $\mathfrak{D}_{\mathbb{A}, T}$ can be naturally identified with a subring of $\mathfrak{A}_{\mathbb{A}}$, and the induced topology is in fact the product topology of $\mathfrak{D}_{\mathbb{A}, T}$.

An order can be recovered from its adelization $\mathfrak{D}_{\mathbb{A}, \infty(K)}$ by the formula $\mathfrak{D}=\mathfrak{D}_{\mathbb{A}, \infty(K)} \cap \mathfrak{A}$, where $\mathfrak{A}$ embeds diagonally into $\mathfrak{A}_{\mathbb{A}}$. More generally, for any finite set $T$ with $\infty(K) \subseteq T \subseteq \Pi(K)$, the intersection $\mathfrak{D}^{T}=\mathfrak{D}_{\mathbb{A}, T} \cap \mathfrak{A}$ is the $T$-order obtained from $\mathfrak{D}$ by inverting the non-archimedean places in $T$. Furthermore, conjugation induces a well defined action of $\mathfrak{A}_{\mathbb{A}}^{*}$, on the set of orders of maximal rank, satisfying $\left(a \mathfrak{D} a^{-1}\right)_{\mathbb{A}, T}=a \mathfrak{D}_{\mathbb{A}, T} a^{-1}$, and therefore, $\left(a \mathfrak{D} a^{-1}\right)^{T}=a\left(\mathfrak{D}^{T}\right) a^{-1}$ for every finite set $T$ satisfying $\infty(K) \subseteq T \subseteq \Pi(K)$ [3]. Two orders $\mathfrak{D}$ and $\mathfrak{D}^{\prime}$ of maximal rank in $\mathfrak{A}$ are in the same genus if and only if they are in the same orbit for this action, i.e., $\mathfrak{D}^{\prime}=a \mathfrak{D} a^{-1}$ for some $a \in \mathfrak{A}_{\mathbb{A}}$. They are in the same spinor genus when $a$ can be chosen as $a=b c$ where $b \in \mathfrak{A}$, diagonally embedded into $\mathfrak{A}_{\mathbb{A}}$, while every local coordinate $c_{\wp}$ of $c$ has trivial reduced norm. Genera and spinor genera for $T$-orders are defined analogously. When $\mathfrak{A}$ satisfies Eichler's condition, every spinor genus contains a unique conjugacy class. This is not the case for definite quaternion algebras over the rationals, which are the ones that interest us in this work. Nevertheless, spinor genera still play a role in the general setting $(\S 3)$.

\section{SPINOR Class Fields AND Classifying GRAPHS}

For any genus $\mathbb{O}=\operatorname{Gen}(\mathfrak{D})$ of orders of maximal rank, the spinor class field $\Sigma=\Sigma(\mathbb{O})$ is the class field corresponding to the class group $K^{*} H(\mathfrak{D}) \subseteq J_{K}=\mathbb{A}^{*}$, where the spinor image $H(\mathfrak{D})$ is defined by

$$
H(\mathfrak{D})=\left\{N(a) \mid a \in \mathfrak{A}_{\mathbb{A}}^{*}, \mathfrak{D}=a \mathfrak{D} a^{-1}\right\},
$$

where $N: \mathfrak{A}_{\mathbb{A}}^{*} \rightarrow J_{K}$ is the reduced norm on adeles. There is a well defined distance map $\rho: \mathbb{O} \times \mathbb{O} \rightarrow \operatorname{Gal}(\Sigma / K)$, satisfying $\rho\left(\mathfrak{D}, a \mathfrak{D} a^{-1}\right)=$ $[N(a), \Sigma / K]$, for any adelic element $a \in \mathfrak{A}_{\mathbb{A}}^{*}$, where $x \mapsto[x, \Sigma / K]$ is the Artin map on ideles. Two orders $\mathfrak{D}$ and $\mathfrak{D}^{\prime}$ are in the same spinor genus if and only if $\rho\left(\mathfrak{D}, \mathfrak{D}^{\prime}\right)=\mathrm{id}_{\Sigma}$.

More generally, for every finite set of places $T$ satisfying $\infty(K) \subseteq$ $T \subseteq \Pi(K)$, and for every genus of $T$-orders, we can define a spinor class field as above. In fact, for the genus $\mathbb{O}^{T}=\left\{\mathfrak{D}^{T} \mid \mathfrak{D} \in \mathbb{O}\right\}$, the 
corresponding spinor class field $\Sigma^{T}$ is the largest subfield of $\Sigma=\Sigma(\mathbb{O})$ splitting completely at every non-archimedean place in $T$, since the reduced norm is surjective at non-archimedean places.

Let $T=\infty(K) \cup\{\wp\}$ for some place $\wp$ splitting $\mathfrak{A}$. Then $\mathfrak{A}$ satisfies Eichler's condition for the set $T$, so that every spinor genus of $T$-orders contains a unique conjugacy class. We conclude that, if $\mathfrak{D}_{1}$ and $\mathfrak{D}_{2}$ are in the same spinor genus, then the $T$-orders $\mathfrak{D}_{1}^{T}$ and $\mathfrak{D}_{2}^{T}$ are conjugate. Replacing $\mathfrak{D}_{2}$ by a conjugate if needed, we can assume that $\mathfrak{D}_{1}^{T}=\mathfrak{D}_{2}^{T}$. In this case, $\mathfrak{D}_{1}$ and $\mathfrak{D}_{2}$ are conjugate if and only if there is an element $a \in \mathfrak{A}$ satisfying both, $a \mathfrak{D}_{1}^{T} a^{-1}=\mathfrak{D}_{1}^{T}$ and $a \mathfrak{D}_{1, \wp}^{T} a^{-1}=\mathfrak{D}_{2, \wp}$. We conclude that conjugacy classes of orders $\mathfrak{H}$, where $\mathfrak{H}^{T}$ is conjugate to $\mathfrak{D}^{T}$ are in one to one correspondence with the orbits of the conjugacy stabilizer $\Gamma=\operatorname{stab}_{\mathfrak{A}^{*}}\left(\mathfrak{D}^{T}\right)$ on the set of orders of maximal rank in $\mathfrak{A}_{\wp}$ that are conjugate to $\mathfrak{D}_{\wp}$.

When $\mathfrak{D}_{\wp}$ is maximal, the latter set is simply the set $\mathbb{O}_{\wp}$ of maximal orders in $\mathfrak{A}_{\wp}$. We can identify $\mathbb{O}_{\wp}$ with the set of vertices of the local Bruhat-Tits tree $\mathfrak{T}_{\wp}$ for $\mathrm{PSL}_{2}\left(K_{\wp}\right)$ [20, $\S$ II.2]. In particular, the set of conjugacy classes of orders $\mathfrak{H}$, where $\mathfrak{H}^{T}$ is conjugate to $\mathfrak{D}^{T}$ is in one to one correspondence with the set of vertices of the quotient graph (in the sense of Serre [19], see the remark bellow) $\Gamma \backslash \mathfrak{T}_{\wp}$. We call $\Gamma \backslash \mathfrak{T}_{\wp}$ the classifying graph of $\mathfrak{D}$ at $\wp$. Note that, when $\mathfrak{D}_{\wp}$ and $\mathfrak{D}_{\wp}^{\prime}$ are two neighbor in the tree, then $\mathfrak{D}_{\wp}^{\prime}=a \mathfrak{D}_{\wp} a^{-1}$ for some local element $a \in \mathfrak{A}_{\wp}$ whose reduced norm is a uniformizing parameter $\pi_{\wp}$. In particular, if $\mathfrak{D}_{1}$ and $\mathfrak{D}_{2}$ are maximal orders corresponding to neighboring vertices in the tree, their distance satisfies $\rho\left(\mathfrak{D}_{1}, \mathfrak{D}_{2}\right)=[e(\wp), \Sigma / K]$, where $e(\wp)$ is an idele whose coordinates are as follows:

$$
e(\wp)_{\mathfrak{q}}=\left\{\begin{array}{rll}
\pi_{\wp} & \text { if } \quad \mathfrak{q}=\wp \\
1 & \text { if } \quad \mathfrak{q} \neq \wp
\end{array}\right.
$$

We conclude that the orders corresponding to the vertices in the classifying graph of $\mathfrak{D}$ belong to a unique spinor genus if $\wp$ splits in $\Sigma / K$ and two spinor genera otherwise. In the latter case the classifying graph is bipartite, so in particular, it contains no loops.

Remark. The definition of quotient graph given in [19] assumes that the group acts on the tree without inversion. This can be fixed, as noted in the same reference, by replacing the tree by its first barycentric subdivision. We adopt this convention in all that follows, but we reserve the word vertex for a vertex of the original graph, while the barycenters of edges are called virtual vertices. When drawing actual pictures, virtual vertices are not drawn unless they are endpoints, i.e., their valency is 1 (See Fig. 1). This type of endpoints appear only if the original action had inversions. 


\section{$(\mathrm{A}) \bullet *$}

Figure 1. A vertex $(\bullet)$ with an edge ending on a virtual endpoint $(*)$.

Remark. We should note that the quotient graph defined here have been applied in existing literature to the study of representation of commutative orders. See for example [17].

\section{Characterizing the orders With Cubic Roots}

Next result gives a complete characterization of the orders in a genus containing a cubic root of unity. This will be used in next section to prove our main theorems.

Proposition 4.1. Let $\mathbb{O}$ be a genus of orders of maximal rank in the definite rational quaternion algebra $\mathfrak{A}$ containing a cubic root of unity. Assume the following conditions hold:

- $\mathfrak{A}_{2} \cong \mathbb{M}_{2}\left(\mathbb{Q}_{2}\right)$.

- The orders in $\mathbb{O}$ are maximal at 2.

Let $\mathfrak{G}=\Gamma \backslash \mathfrak{T}_{2}$ be the classifying graph at 2, as described in the preceding section. Then the orders orders in $\mathbb{O}$ containing a cubic root of unity correspond precisely to the (non-virtual) endpoints, i.e., vertices of valency one, in $\mathfrak{G}$.

Proof. Let $\mathfrak{D} \in \mathbb{O}$ be an order containing a cubic root of unity $\omega$. Since $\mathbb{Z}_{2}[\omega]$ is the ring of integers in the unique unramified quadratic extension of $\mathbb{Q}_{2}$, then $\mathbb{Z}_{2}[\omega]$ is contained in a unique maximal order of $\mathfrak{A}_{2}$ [5, Prop. 4.2], namely $\mathfrak{D}_{2}$. Recall that the conjugation-stabilizer of a local maximal order $\mathfrak{E}_{2}$, is $K^{*} \mathfrak{E}_{2}^{*}$. Since $\omega$ is a unit we conclude that conjugation by $\omega$ permutes transitively the three neighbors of $\mathfrak{D}$, and therefore the vertex in $\mathfrak{G}$ corresponding to $\mathfrak{D}$ has valency one.

Now let $\mathfrak{D}$ be a global order corresponding to a vertex of valency one in the classifying graph $\mathfrak{G}$. Let $\Gamma_{\mathfrak{D}}$ be the stabilizer of $\mathfrak{D}$ in $\Gamma$. Then $\overline{\Gamma_{\mathfrak{D}}}=\Gamma_{\mathfrak{D}} / K^{*}$ is the automorphism group of the order $\mathfrak{D}$, and therefore it is finite, since $\mathfrak{A}$ is definite. Since $\mathfrak{D}$ corresponds to a vertex of valency one in $\mathfrak{G}$, the group $\overline{\Gamma_{\mathfrak{D}}}$ must permute transitively the three neighbors of $\mathfrak{D}$, and therefore it contains an element of order 3 . In other words, there exists an element $u \in \mathfrak{A} \backslash K$ satisfying $u \mathfrak{D} u^{-1}=\mathfrak{D}$ and $u^{3} \in K^{*}$. This element has a quadratic minimal polynomial with rational (and therefore real) coefficients. So it has either two real roots or two conjugate complex roots. 
Now consider $u$ as an element of $\mathfrak{A}_{\overline{\mathbb{Q}}}=\mathfrak{A} \otimes_{\mathbb{Q}} \overline{\mathbb{Q}} \cong \mathbb{M}_{2}(\overline{\mathbb{Q}})$, where $\overline{\mathbb{Q}}$ is the field of algebraic numbers. Then $u$ is conjugate to a real multiple of a matrix of the form $\left(\begin{array}{cc}\eta_{1} & 0 \\ 0 & \eta_{2}\end{array}\right)$ where $\eta_{1}$ and $\eta_{2}$ are two different cubic roots of unity. By the previous discussion we can assume either $\left(\eta_{1}, \eta_{2}\right)=\left(\omega, \omega^{2}\right)$ or $\left(\eta_{2}, \eta_{1}\right)=\left(\omega, \omega^{2}\right)$. Hence, the projective representation $\rho: C_{3} \rightarrow \mathfrak{A}^{*} / K^{*}$ of the cyclic group $C_{3}$ defined by $u$ is conjugated to the representation defined by a cubic root of unity over $\overline{\mathbb{Q}}$, and therefore also over $\mathbb{Q}$ by $[2$, Th. 1$]$, so we can assume that $u$ is a root of unity. Since the stabilizer of a local maximal order $\mathfrak{D}_{\wp}$ equals $K_{\wp}^{*} \mathfrak{D}_{\wp}^{*}$ at split places, and $\mathfrak{D}_{\wp}$ contains all local integers at ramified places, we must conclude that $u \in \mathfrak{D}^{*}$, locally everywhere and hence globally. The result follows.

Example. Consider the quaternion algebra $\mathfrak{A}=\left(\frac{-3,-3}{\mathbb{Q}}\right)$. Let $i$ and $j$ be generators satisfying $i^{2}=j^{2}=-3$ and $i j=-j i$. Set $i=2 \eta+1$ and $j=2 \omega+1$, so that $\eta$ and $\omega$ are cubic roots of unity. Then each of the orders $\mathfrak{D}=\mathbb{Z}[\eta, j]$ and $\mathfrak{D}^{\prime}=\mathbb{Z}[i, \omega]$ is contained in a unique maximal order, since they are maximal outside the place 3 and $\mathfrak{A}_{3}$ has a unique maximal order. Denote these maximal orders by $\mathfrak{D}_{0}$ and $\mathfrak{D}_{0}^{\prime}$. They are endpoints of the Classifying Graph at 2 by the previous proposition. We claim that they are neighbors. Since they are also isomorphic, and therefore conjugate, we must conclude that all maximal orders in $\mathfrak{A}$ are conjugate.

Now we prove the claim. It follow from the tables in [18] that $\mathbb{Z}_{2}[i, j]$ is contained in exactly 2 maximal orders. Alternatively, we can observe that $\mathbb{Z}_{2}[i+j] \cong \mathbb{Z}_{2}[\sqrt{-6}]$ is the ring of integers of a ramified extension, and therefore it is contained in exactly 2 maximal orders by [ 5 , Prop. $4.2]$.

\section{Proofs of the MAIN Results}

Theorem 1 follows from proposition 4.1 and Proposition 5.1 below:

Proposition 5.1. Let $G$ be a connected graph where every vertex has valency 3 or less. If $G$ has $n=r+t$ vertices, $r$ of which have valency 1 , then $r \leq t+2$, or equivalently, $r \leq \frac{n}{2}+1$. Equality, in both inequalities, holds if and only if $G$ is a tree where every vertex have valency 1 or 3.

Proof. Since the graph is connected, the number of edges is $v \geq n-1$, with equality if and only if the graph is a tree. On the other hand, since every edge has two endpoints, $3 t+r \geq 2 v$. We conclude that $3 t+r \geq 2(t+r-1)$, with equality if and only if each of the previous inequalities is an equality. The result follows. 
Remark. Note that we might not have equality in Theorem 1.1 even if the quotient graph satisfy the sufficient conditions for equality in Proposition 5.1 above, because of the existence of virtual endpoints. This can be used in a few cases to improve the bound $r \leq t+2$ and, a fortiori, also $r \leq \frac{n}{2}+1$. In fact, the presence of some particular subfields imply the existence of virtual endpoints. See the examples in the final section.

Next result is needed in the proof of Theorem 2.

Proposition 5.2. Let $G$ be a bipartite graph with a set of vertices $V(G)=A \cup B$, where every vertex of $G$ joins a vertex in $A$ and $a$ vertex in $B$. Let $n$ be the cardinality of $B$, while $r$ is the number of endpoints in $B$. If no vertex of $G$ has a valency larger than 3 , then $r \leq 3(n+1) / 4$. Equality holds if and only if the following conditions hold:

(1) $G$ is a tree.

(2) Every vertex in $A$ has valency 3.

(3) Every vertex in $B$ has valency 3 or 1.

Proof. First we assume that $G$ satisfies conditions (1)-(3). Let $t=n-r$ as before. Let $m, p$, and $s$ the numbers of vertices in $A$ with zero, one, or two neighboring endpoints, respectively. Then the previous result shows that $r=t+m+p+s+2$, while on the other hand, we have the identities $r=p+2 s$ and $3 t=3 m+2 p+s$. Adding this two identities, we get $r+3 t=3(m+p+s)=3(r-t-2)$, whence $r=3(t+1)$. Replacing $t$ by $n-r$, the result follows.

We call nails and forks to subgraphs of the shapes shown in Figure 1(A). Now, for the general case, we observe that, by adding extra nails and forks, as shown in Figure 1(C), we can turn any tree in a tree satisfying the preceding hypotheses. Furthermore, cycles can be eliminated by replacing an edge by a nail-fork pair, as shown in Figure 1(B). By repeated application of these procedures, we obtain a graph $G^{\prime}$ satisfying the hypotheses. Set $V\left(G^{\prime}\right)=A^{\prime} \cup B^{\prime}$ as before, where $B$ is identified with a subset of $B^{\prime}$ in an obvious way. Let $n^{\prime}$ be the cardinality of $B^{\prime}$, while $r^{\prime}$ is the number of endpoints in $B^{\prime}$, and $t^{\prime}=n^{\prime}-r^{\prime}$. Note that $t^{\prime}=t$ and $r \leq r^{\prime}$, by a case by case inspection of the reduction steps in Figure 1. We conclude that

$$
r \leq r^{\prime}=3\left(t^{\prime}+1\right)=3(t+1)=3(n+1)-3 r,
$$

whence the result follows. Furthermore, the inequality is strict unless no reduction was needed. 


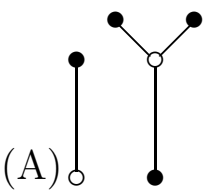

(B)

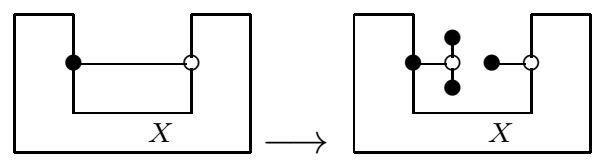

(C)

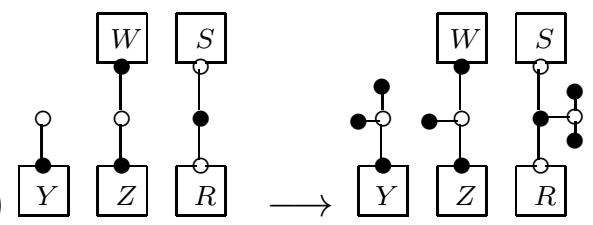

Figure 2. (A) Nail and fork. (B) Deleting a cycle by introducing a nail-fork pair. (C) Adding nails and forks to several trees.

Proof of Theorem 2. Let $\mathfrak{D}$ be an order in the spinor genus $\mathbb{O}$, and let $\Sigma$ be the spinor class field corresponding to this genus. Let $e(2) \in J_{\mathbb{Q}}$ be the idele whose only non-trivial coordinate is $e(2)_{2}=2$, and let $\sigma=[e(2), \Sigma / \mathbb{Q}]$. If $\sigma$ is the identity, the vertices in the quotient graph $\mathfrak{G}$ correspond to maximal orders in one spinor genus and the result follows from Proposition 5.1 since $\frac{n}{2}+1 \leq \frac{3}{4}(n+1)$ for $n \geq 1$. If $\sigma$ is not the identity, the graph $\mathfrak{G}$ is bipartite, and its vertices belong to exactly two spinor genera, so the result follows from Proposition 5.2 .

Proof of Theorem 3. Assume all conditions in the theorem hold, and let $\sigma$ be as in the preceding proof. If $\sigma$ is the identity, the vertices in $\mathfrak{G}$ correspond to maximal orders in one spinor genus and the result follows from Proposition 5.1 as before. We assume therefore that $\sigma$ is not the identity, so in particular the graph $\mathfrak{G}$ is bipartite, with any pair of adjacent vertices in different spinor genera. Let $\mathbb{O}$ and $\mathbb{O}_{1}$ be such spinor genera. Since $\mathbb{Z}[\omega]$ is not spinor selective for this genus, there must exists an order in each spinor genus containing a copy of $\mathbb{Z}[\omega]$. Let $\mathfrak{D}_{1} \in \mathbb{O}_{1}$ be such an order. Let $\mathfrak{D} \in \mathbb{O}$ be a neighbor of $\mathfrak{D}_{1}$. If $\mathbb{Z}[\omega]$ is not selective for the spinor genus $\mathbb{O}$, both $\mathfrak{D}$ and $\mathfrak{D}_{1}$ are endpoints and the graph contains only two vertices, a contradiction.

\section{Generalizations and examples}

The methods employed here for the ring $\mathbb{Z}[\omega]$ works with a limited number of orders whose presence is reflected on the combinatorial properties of the local graph at some finite place $\wp$. 
Example. Roots of unity $\eta$ whose order is a Fermat prime $p=2^{2^{n}}+1$ produce vertices of valency 1 for any definite quaternion algebra defined over the totally real field $L=\mathbb{Q}(\eta) \cap \mathbb{R}$, at any dyadic place $\wp$ of $L$. This can be proved as follows:

The extension $\mathbb{Q}(\eta) / \mathbb{Q}$ is unramified at 2 , since the polynomial $x^{p}-1$ has different roots in $\overline{\mathbb{F}_{2}}$, while each of them generates an extension of degree $2^{n}+1$ over $\mathbb{F}_{2}$, as $m=2^{n}+1$ is the smallest value of $m$ for which $p$ divides $2^{m}-1$. Furthermore, the quadratic extension $\mathbb{Q}(\eta) / L$ is inert at every dyadic place, since $\eta \mapsto \eta^{-1}$ induces a non-trivial isomorphism over the residue field. In particular, for every dyadic place $\wp$ of $L$, the residue field $\mathbb{L}$ satisfies $\left[\mathbb{L}: \mathbb{F}_{2}\right]=2^{n}$. We conclude that the local Bruhat-Tits tree for $\mathrm{PSL}_{2}\left(L_{\wp}\right)$ at any such place has vertices of valency $2^{2^{n}}+1=p$. Now the result follows as in the proof of Proposition 4.1.

As a consequence, we conclude, as in the proof of Theorem 1.2 that the number $r$ of conjugacy classes, in any spinor genus of $\mathcal{O}_{L}$-orders of size $m$, containing a unit of order $p=2^{2^{n}}+1$, satisfies

$$
r \leq \frac{p(p-2)}{(p-1)^{2}}\left(m+\frac{1}{p-2}\right) .
$$

This again shows that $\mathcal{O}_{L}[\eta]$ is selective for large values of $m$.

Example. Let $\mathfrak{A}$ be a definite quaternion algebra splitting at 2 . Consider the commutative order $\mathbb{Z}[u]=\mathcal{O}_{\mathbb{Q}[u]}$, where $u$ is a root of the equation $x^{2}-x+2=0$. Note that the extension $\mathbb{Q}[u] / \mathbb{Q}$ splits at 2 , whence the local orders at 2 containing a fixed copy of $\mathbb{Z}_{2}[u]$ in $\mathfrak{A}_{2}$ lie in a maximal path $\gamma$ in the Bruhat-Tits tree [5, Prop. 4.2]. Since the norm of $u$ is a uniformizing parameter, it is easy to see that, for any global embedding $\phi: \mathbb{Q}[u] \rightarrow \mathfrak{A}$, conjugation by $\phi(u)$ defines a global automorphism of $\mathfrak{A}$ that shifts by 1 the line of local orders containing $\phi(u)$. Since $u$ is a unit outside of 2 , conjugation by $\phi(u)$ must stabilize any $\mathbb{Z}[1 / 2]$-order $\mathfrak{D}$ containing it. It follows that all orders in $\gamma$ are isomorphic and are connected to equivalent branches of the tree (Figure $3 \mathrm{~A})$. We conclude that the corresponding vertex in the quotient graph looks like one of the graphs in Figure 3B, according to whether there exist a global inversion on this path or not. It follows that the proof of Theorems 1.1-1.3 carry over word by word to this case.

Example. When $K$ is the function field of a smooth projective curve $X$ over a finite field $\mathbb{F}$, quotient graphs similar to those described here can be used to classify $X$-orders in a quaternion $K$-algebra $\mathfrak{A}[\underline{6}$, Thm. 


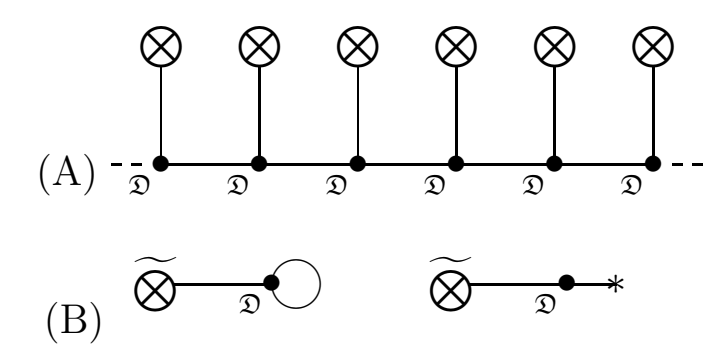

Figure 3. (A) Repetitive pattern arising from a global uniformizing parameter in a field splitting at 2. (B) Possible shapes of the quotient graph. Here $\widetilde{\otimes}$ denotes the image of the subgraph $\bigotimes$.

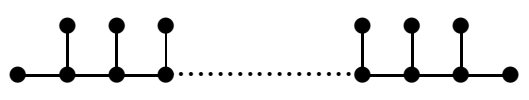

Figure 4. The classifying graph of a conjectural large genus for which $\mathbb{Z}[\sqrt{-3}]$ would not be selective.

1.1]. These graphs are finite if and only if $\mathfrak{A}$ is a division algebra. They depend on the choice of a place at infinity, playing the same role as the place 2 in our case. If the place at infinity is defined over $\mathbb{F}$, endpoints correspond to orders representing the maximal order of the subfield $K \mathbb{L}$ where $\mathbb{L}$ is the unique quadratic extension of $\mathbb{F}[6$, Ex. 5.1]. The same argument given here can be used to show that this order is selective on large spinor genera.

Example. It follows easily from [5, Prop. 2.4] that if the algebra $\mathfrak{A}$ contains a cubic root of unity and if $r$ is the largest distance of a vertex from the set of non-virtual endpoints, in the classifying graph at 2 of maximal orders in $\mathfrak{A}$, then $\mathfrak{H}=\mathbb{Z}+2^{r} \mathbb{Z}[\omega]=\mathbb{Z}\left[2^{r-1} \sqrt{-3}\right]$ is contained in every maximal order in $\mathfrak{A}$. The methods in this work cannot be used to prove selectivity for the order $\mathbb{Z}[\sqrt{-3}]$, since it is easy to draw graphs where every vertex is at distance 1 from an endpoint, as in Figure 4. Whether these graphs can actually be classifying graphs for some family of spinor genera is still unknown to us. If such genera existed we would have an example of an order that is non-selective for arbitrarily large spinor genera.

By the theorem of almost strong approximation [13, Th. 3.1], the order $\mathbb{Z}\left[p^{2} \sqrt{-d}\right]$ is not selective in a fixed genus, for almost every prime 
$p$, since this result implies that every pair of maximal orders are at most at a distance 2 in the corresponding graph. Furthermore, the main result in [10] states:

Any positive definite ternary quadratic lattice of discriminant $d$ represents any integer of the form $c t^{2}$, if $t$ is large enough, $(t, 2 d)=1$, and $c$ is primitively represented by the corresponding genus.

This certainly implies a stronger version of the previous statements. All these results refer to large factors, however, so the following question remains open, as far as we know:

Is any order in a imaginary quadratic field selective for any large enough spinor genus?

As noted above, if the answer to this question is positive, this has significant implications in the possible shapes of the classifying graphs.

Let $\mathfrak{A}(p)$ be the unique quaternion algebra over $\mathbb{Q}$ that ramifies exactly at the places $p$ and $\infty$. We end this work by computing the quotient graph at 2 of the genus of maximal orders for all odd primes for which the class number of the algebra $\mathfrak{A}(p)$ is 1 , that is when $p \in\{3,5,7,13\}$, cf. [14, §3].

When $\mathbb{Q}[\omega]$ embeds into $\mathfrak{A}(p)$, namely, when $p \in\{3,5\}$, the unique vertex of the classifying graph has valency 1 , an therefore the graph looks like Figure $5 \mathrm{~A}$. Since $\mathbb{Q}[\sqrt{-7}]$ embeds into $\mathfrak{A}(7)$, it follows from the second example in this section that the classifying graph $\Gamma \backslash \mathfrak{T}_{2}$ has one of the shapes in Figure 3B. Note that $\mathfrak{A}(7)=\left(\frac{-7,-1}{\mathbb{Q}}\right)$, whence for any embedding $\phi: \mathbb{Q}[u] \rightarrow \mathfrak{A}(7)$, where $u=\frac{\sqrt{-7}+1}{2}$, there in a pure quaternion $i \in \mathfrak{A}(7)$ satisfying $i^{2}=-1$ and $i \phi(u) i^{-1}=\overline{\phi(u)}$, whence conjugation by $i$ permutes the eigenvectors of $\phi(u)$. Since conjugation by $\phi(u)$ is a shift in the path $\gamma$ of maximal orders containing $\phi(u)$, as in Figure 3A, conjugation by $i$ is an inversion on that line. We conclude that $\Gamma \backslash \mathfrak{T}_{2}$ looks like Figure 5B. The same holds for $p=13$ if we note that $\mathfrak{A}(13)=\left(\frac{-7,-13}{\mathbb{Q}}\right)$, and any pure quaternion satisfying $i^{2}=-13$ stabilizes any maximal $\{\infty, 2\}$-order containing it, even if $i$ is not a $\{\infty, 2\}$-unit, since the maximal order at 13 is unique, and $N(i)$ is a unit outside 13. The result follows.

\section{REFERENCES}

[1] L. Arenas-Carmona, Applications of spinor class fields: embeddings of orders and quaternionic lattices, Ann. Inst. Fourier 53 (2003), 2021-2038. 
(A)

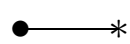

(B)

Figure 5. Classifying graphs for the genus of maximal orders at 2 for the algebra $\mathfrak{A}(p)$ when $(\mathrm{A}) p \in\{3,5\}$. (B) $p \in\{7,13\}$.

[2] L. Arenas-Carmona, Projective representations in algebras and cohomology, Arch. Math. 97 (2011), 105-113.

[3] L. Arenas-Carmona. Representation fields for commutative orders, Ann. Inst. Fourier 62 (2012), 807-819.

[4] L. Arenas-Carmona, Maximal selectivity for orders in fields, J. Number T. 132, (2012), 2748-2755.

[5] L. Arenas-Carmona, Eichler orders, trees and Representation Fields, Int. J. Number Th. 9 (2013), 1725-1741.

[6] L. Arenas-Carmona, Computing quaternion quotient graphs via representations of orders, J. Algebra 402 (2014), 258-279.

[7] W.K. ChAN and F. XU, On representations of spinor genera, Compositio Math. 140.2 (2004), 287-300.

[8] C. Chevalley, L'arithmétique sur les algèbres de matrices, Herman, Paris, 1936.

[9] M. Deuring, Die Anzahl der Typen von Maximalordungen einer definiten Quaternionenalgebra mit primer Grundzahl, Jahresbericht der Deutschen Mathematiker-Vereinigung 54 (1951), 24-41

[10] A.G. EARNEST, On the representation of integers with large square factors by positive define ternary quadratic forms, Mathematika 31.2 (1984) 252-257.

[11] T. Chinburg and E. Friedman, An embedding theorem for quaternion algebras, J. London Math. Soc. 60.2 (1999), 33-44.

[12] X. GuO and H. QIN, An embedding theorem for Eichler orders, J. Number Theory 107 (2004), 207-214.

[13] J.S. Hsia and M. Jöchner, Almost strong approximations for definite quadratic spaces, Invent. Math. 129 (1997) 471-487.

[14] J.-I. IGusA, Class number of a definite quaternion with prime discriminant Proc. Natl. Acad. Sci. USA. 44.4 (1958) 312-314.

[15] B. Linowitz, Selectivity in quaternion algebras, J. Number Theory 132 (2012), 1425-1437.

[16] C. MaclaChlan, Optimal embeddings in quaternion algebras, J. Number Theory 128 (2008), 2852-2860.

[17] I. PAYs, Arbres, ordres maximaux et formes quadratiques entières, London Math. Soc. Lectures Note Ser. 215, Cambridge Univ. Press., Cambridge (1995) 209-230.

[18] I. SaAVedra, Cálculos explícitos de la imagen espinorial local relativa para ordenes cíclicos, Master Thesis, Universidad de Chile, Santiago, 2014.

[19] J.-P. Serre, Trees, Springer Verlag, Berlin, 1980.

[20] M.-F. Vigneras, Arithmétique des algèbres de Quaternions, Springer Verlag, Berlin, 1980. 